\title{
A cryogenic scanning laser microscope for investigation of dynamical states in long Josephson junctions
}

Holm, Jesper; Mygind, Jesper

Published in:

I E E E Transactions on Applied Superconductivity

Link to article, DOI:

10.1109/77.403159

Publication date:

1995

Document Version

Publisher's PDF, also known as Version of record

Link back to DTU Orbit

Citation (APA):

Holm, J., \& Mygind, J. (1995). A cryogenic scanning laser microscope for investigation of dynamical states in long Josephson junctions. I E E E Transactions on Applied Superconductivity, 5(2), 2747-2750.

https://doi.org/10.1109/77.403159

\section{General rights}

Copyright and moral rights for the publications made accessible in the public portal are retained by the authors and/or other copyright owners and it is a condition of accessing publications that users recognise and abide by the legal requirements associated with these rights.

- Users may download and print one copy of any publication from the public portal for the purpose of private study or research.

- You may not further distribute the material or use it for any profit-making activity or commercial gain

- You may freely distribute the URL identifying the publication in the public portal

If you believe that this document breaches copyright please contact us providing details, and we will remove access to the work immediately and investigate your claim. 


\title{
A Cryogenic Scanning Laser Microscope for Investigation of Dynamical States in Long Josephson Junctions
}

\author{
Jesper Holm and Jesper Mygind \\ Physics Department, Technical University of Denmark, B309, DK-2800 Lyngby, Denmark \\ and \\ Danish Institute of Fundamental Metrology, B307, DK-2800 Lyngby, Denmark
}

\begin{abstract}
The first local oscillators based on moving magnetic flux quanta in long Josephson junctions are being developed for superconducting integrated quasi-optical SIS receivers. In order to further refine these oscillators one has to understand the complex dynamics of these devices. Since the local tunnel current is one of the most important internal junction parameters which together with the boundary conditions determine the dynamics, it is of vital importance to experimentally determine the current density throughout the entire junction with high spatial resolution. Here we report on measurements on different oscillator samples, performed with a novel Cryogenic Scanning Laser Microscope (CSLM) having a spatial resolution of less than $\pm 2.5 \mu \mathrm{m}$ over a $500 \mu \mathrm{m} \times$ $50 \mu \mathrm{m}$ wide scanning area in the temperature range $2 \mathrm{~K}-300 \mathrm{~K}$. Even though the dynamical states are extremely sensitive to external noise this microscope enables us to make stable in-situ measurements on operating Josephson junctions. Recent results are presented and discussed.
\end{abstract}

\section{INTRODUCTION}

The interest in developing scanning electron and scanning laser microscopes for spatially resolved investigations of various semi- and superconducting devices at cryogenic temperatures has increased during the last few years[1]-[3]. Due to recent improvements in the fabrication technique of such devices, it has become increasingly difficult to combine the requirements for high resolution and high mechanical stability with the demands for low noise environments, large scanning area, and robust and easy-to-operate overall design.

We have designed a cryogenic scanning laser microscope (CSLM)[4], which to a large extend meets these requirements, and used it in experiments on a series of chips with various superconducting Josephson junction circuits.

The CSLM relies on the detection of the electrical response of the circuit to a very localized heating induced by irradiation with $675 \mathrm{~nm}$ wavelength light from a Al-

Manuscript received October 18, 1994.

J. Holm, e-mail: jeh@mips.fys.dtu.dk; J. Mygind, e-mail: myg@mips.fys.dtu.dk; fax (+45) 45931669 , phone (+45) 42881611.
GaInP semiconductor laser. The hot-spot is moved by a specially designed piezo-electric scanner sweeping the tip of a single-mode optical fiber a few $\mu \mathrm{m}$ above the circuit.

The piezoelectric scanner, which is specially designed to achieve a relatively large scanning range at low temperatures, consists of two pairs of series coupled bimorph benders, made by two equally sized and oppositely mounted (with respect to the pooling field) piezoelectric plates. A scanning range well above $500 \mu \mathrm{m}$ has been demonstrated at $4.2 \mathrm{~K}$.

In contrast to earlier designs[1], [5]--[8] the sample and the entire microscope including the piezo-scanner holding the glass fiber tip, the sample mounting stage, and the mechanical alignment system is maintained at low temperatures. This offers several advantages: thermal drift and fluctuations are reduced, suppression of mechanical, electrical and magnetic noise is improved, and in situ measurements can be made directly on the chip with the thinfilm circuit in operation.

An elegant use of the controlled local heating is to dragand-drop flux quanta into an annular Josephson junction[9] or a SQUID ring. Practically this is done by moving across the ring the hot-spot enclosing a single flux quantum trapped in the small normal conducting region induced by the laser heating in the superconducting film. In contrast to the electron beam used in [9], the presence of an external magnetic field necessary for this use will not at all influence the laser beam.

Sec. II introduces a simple physical model for CSLM experiments on long Josephson junctions biased in a static state. In Sec. III we present and discuss the results.

\section{THEORY}

When a narrow laser beam heats a superconductor the high energy photons cause a local change in the quasiparticle and phonon distribution as suggested by Rothwarf and Taylor[10] and later extended by Glass and Rogovin[11]. This results in a new local equilibrium characterized by an higher effective temperature which may be obtained from the quasiparticle density using the BCS gap equation. For simplicity we will therefore assume that the only result of the beam irradiation is a rise of the hot-spot temperature.

$1051-8223 / 95 \$ 04.00$ (c) 1995 IEEE 
Depending on the beam intensity, the optical reflection coefficient, and the thermal properties of the sample under investigation the temperature rise is typically several kelvin with a few tens of microwatts of incident laser power. The spatial resolution depends on the beam radius and the thermal healing length of the sample[12], [13] while, with our relatively low scan rates, the influence from the thermal relaxation time can be neglected.

In experiments using a sample with an spatially extended all-niobium Josephson tunnel junction circuit deposited on a silicon chip a resolution as low as $\pm 2 \mu \mathrm{m}$ has been obtained[13]. This has to be compared to the thermal healing length of 1-3 $\mu \mathrm{m}$ commonly used[3] setting an upper limit for the resolution.

Assuming an ideal tunnel junction formed by two identical electrodes with the energy gap $\Delta$, the spatial dependent quasiparticle current is given by[3]

$$
\begin{aligned}
I_{q p}(x, y, T) & =\frac{\sigma_{n}(x, y)}{e} \int_{-\infty}^{\infty} d E \frac{|E|}{\left[E^{2}-\Delta(T)^{2}\right]^{1 / 2}} \\
& \times \frac{|E+e V|}{\left[(E+e V)^{2}-\Delta(T)^{2}\right]^{1 / 2}} \\
& \times[f(E, T)-f(E+e V, T)] \\
& \equiv \frac{\sigma_{n}(x, y)}{e} \mathcal{K}(V, T)
\end{aligned}
$$

where $E$ is the quasiparticle energy, $V$ the applied voltage, $f(E, T)$ the Fermi distribution function at temperature $T$, and $\sigma_{n}$ the normal state tunneling conductance. For short we have defined $\mathcal{K}$ as the integral. If only a small part of the junction is irradiated and the constant-current bias point is at the quasiparticle branch of the $I V$-curve, well below (or above) the gap, we arrive at the following equation in the weak perturbation limit:

$$
\left.\delta V_{q p}(x, y) \simeq \frac{\partial V}{\partial I}\right|_{I=I_{b}} \frac{\sigma_{n}(x, y)}{e} \mathcal{K}_{0}
$$

showing that the amplitude of the response voltage $\delta V_{q p}$ reflects the local tunneling conductance. $\mathcal{K}$ depends only very weakly on the energy gap and is therefore assumed to be constant and equal to $\mathcal{K}_{0}$.

If the bias point is near the gap the integral $\mathcal{K}$ in Eqn. 1 depends on $\Delta$. In ref. [3] the following expression is derived:

$$
\delta I_{q p}(x, y) \simeq \frac{\sigma_{n}}{e} \delta \mathcal{K}\left(V_{b}, x, y\right) .
$$

For small perturbations $\mathcal{K}$ has a sharp peak at $V_{\text {bias }}=$ $2 \Delta / e$, i.e. $\delta I_{q p}$ shows a sharp peak for a bias voltage equal to the local sum-gap voltage. Therefore scanning a long Josephson junction having a spatially imhomogeneous sum-gap only those regions with $V_{\text {bias }}=2 \Delta / e$ yields a large signal. The spatial distribution of the sum-gap inside the junction can therefore be obtained by recording a series of scans at different bias points.

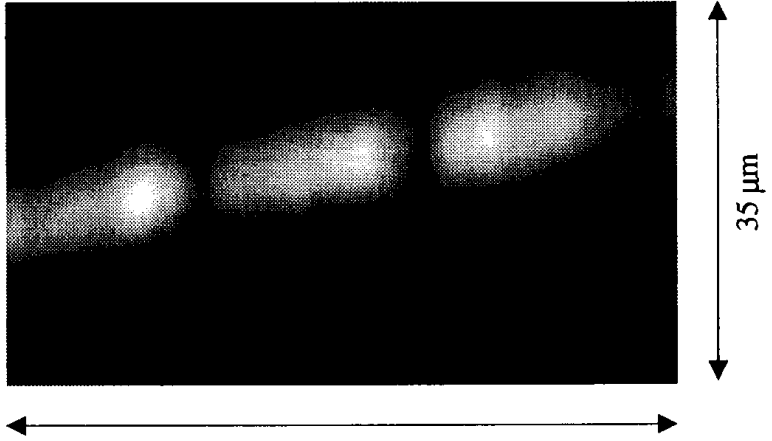

$300 \mu \mathrm{m}$

Fig. 1. Quasiparticle distribution in a part of a $5 \mu \mathrm{m}$ wide inhomogeneous long Josephson tunnel junction. The inhomogeneities are spaced $100 \mu \mathrm{m}$ apart and are $5 \mu \mathrm{m}$ wide. Dark regions correnspond to low voltage response signal, white to maximum signal. The picture shows a sample area of $300 \times 35 \mu \mathrm{m}^{2}$.

\section{Measurements and Discussion}

\section{A. Quasiparticle Distribution}

Fig. 1 shows an image of a part of a $5 \mu \mathrm{m}$ wide and 500 $\mu \mathrm{m}$ long Josephson junction fabricated with a regular lattice of $5 \mu \mathrm{m}$ wide inhomogeneities with a mutual distance of $100 \mu \mathrm{m}$. The voltage response reflects the variation of the quasiparticle tunneling conductance caused by the inhomogeneities as expected from Eqn. (2). Dark regions correspond to minimum response, white to maximum.

The picture, consisting of 256 lines each with 256 sample points, was recorded in $7 \mathrm{~min}$. with a scan-rate of 1.2 lines per second in a 8 bit resolution. Besides demonstrating the resolution of approx. $\pm 2.5 \mu \mathrm{m}$ it also proves the long term stability of the CSLM. The bias point was chosen well below the gap.

\section{B. Gap State Measurements}

Fig. 2 shows the $I V$ curve of a $200 \times 10 \mu \mathrm{m}^{2}$ Josephson junction. Only the quasiparticle curve and the gap state is shown. Because of the rather high return current, it is evident that the tunneling conductance is quite inhomogeneous. This is confirmed by current biasing at point $a$ well below the gap voltage and performing an line-scan along the junction. The resultant voltage is plotted in Fig. 3 as function of the beam position and shows the spatial distribution of the tunneling conductance as discussed above. At the biaspoint $a$ we do see a larger response for a certain beam position inside the junction (marked $\alpha$ ). As we move towards $b$ the response from the inhomogeneity gets stronger compared to the background signal originating from the rest of the junction. The response voltage is now the sum of the response caused by the tunneling conductance distribution and the gap state distribution. 


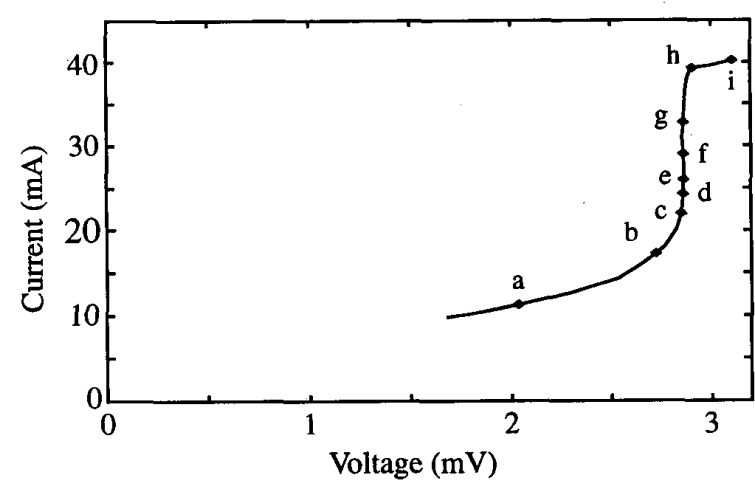

Fig. 2. IV characteristic showing the quasiparticle curve and the gap. At the biaspoints a-i we have performed the line-scans shown in Fig. 3.

At $b$ we observe a splitting of the peak into $\alpha_{1}$ and $\alpha_{2}$. The maximum now corresponds to a point where the local gap voltage is exactly equal to the bias voltage. The splitting is evident at $c$, where another peak (marked $\beta$ ) occurs to the left. This peak again splits up into $\beta_{1}$ and $\beta_{2}$ when we reach $d$. From $e$ to $g$ the peak seems to move towards the edge of the junction reflecting a higher local gap voltage here. It should be noted that the change in bias voltage betweeen $c$ and $g$ is only around $10 \mu \mathrm{V}$. At point $h$, we get a reversed signal from the interior of the junction, indicating that we here are passing the gap. At point $i$ we are well above the gap at a position where the response voltage again reflects the local tunnel current distribution. Having sufficiently many linescans, we are able to plot the spatial distribution of the local gap voltage by simply finding the maximum signal for each line-scan as it also was stated in Ref. [3], but here we have investigated a much simpler geometry. It is evident that positions where we have a larger quasiparticle tunneling correspond to a lovering in the local gap voltage, just as expected from the microscopic theory.

\section{Dynamical States}

The uni-directional viscous flow of magnetic flux quanta in long Josephson tunnel junction with high damping has recently been succesfully used in the development of local oscillators for fully superconducting integrated sub-mm wave SIS receivers[9], [14], [15]. The CSLM has been used to investigate samples containing such flux-flow oscillators (FFO)[16]. Fig. 4a shows a sketch of the $500 \times 5 \mu \mathrm{m}^{2}$ large FFO in the scanned area. Fig. $4 \mathrm{~b}$ shows the voltage response recorded with the FFO junction biased near the gap voltage. The voltage response shown in Fig. 4c is obtained with the junction biased at $V=700 \mu \mathrm{V}$ with an applied magnetic field. From comparing the two pictures one clearly sees a standing wave pattern with period 50

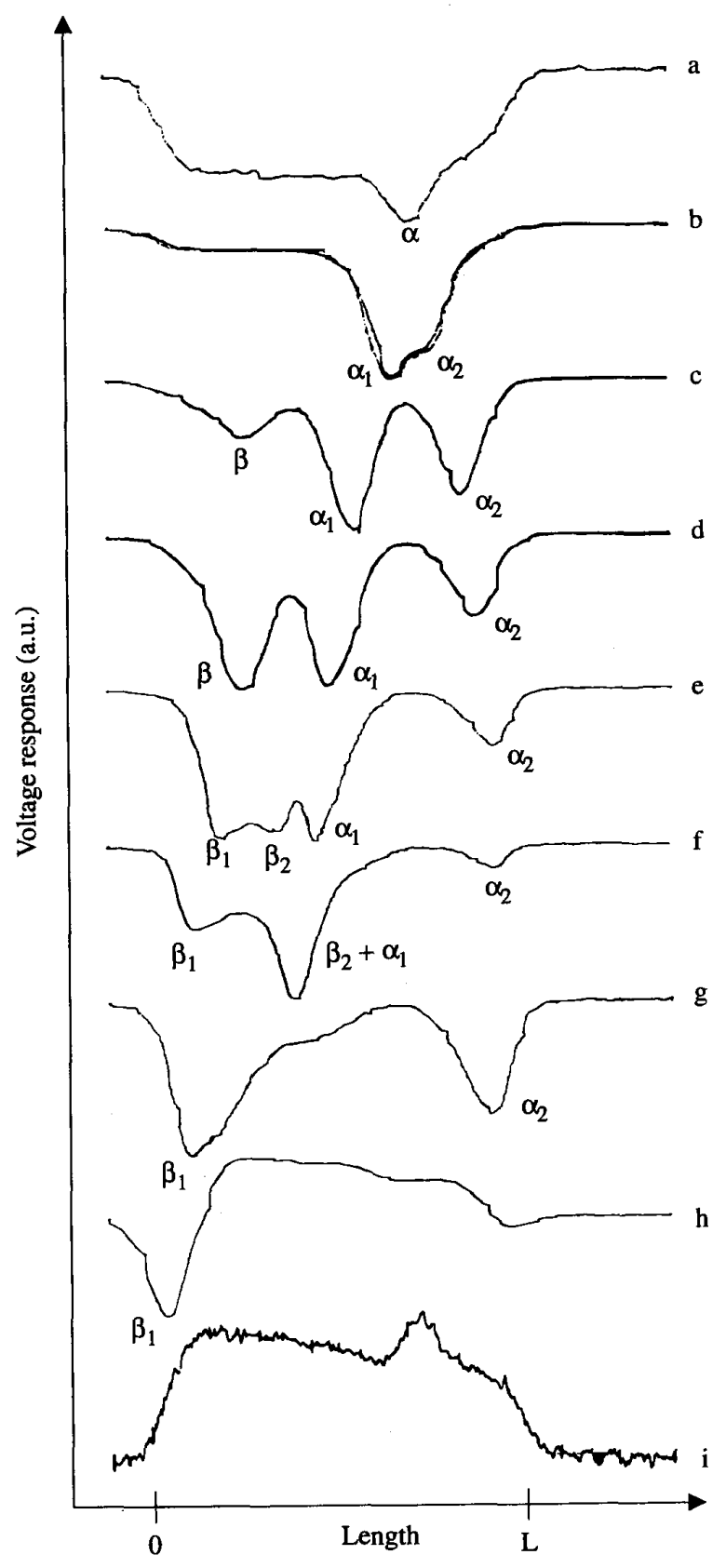

Fig. 3. Line-scans showing the voltage response when biased at the points $a-i$ in Fig. 2. The response voltage is shown as function of the beam position. The line-scans are shown off-set for clarity. Due to slight changes in the amplification, the voltage response for the different biaspoints can only be compared qualitatively. The total scanning range is $300 \mu \mathrm{m}$.

$\mu \mathrm{m}$. 

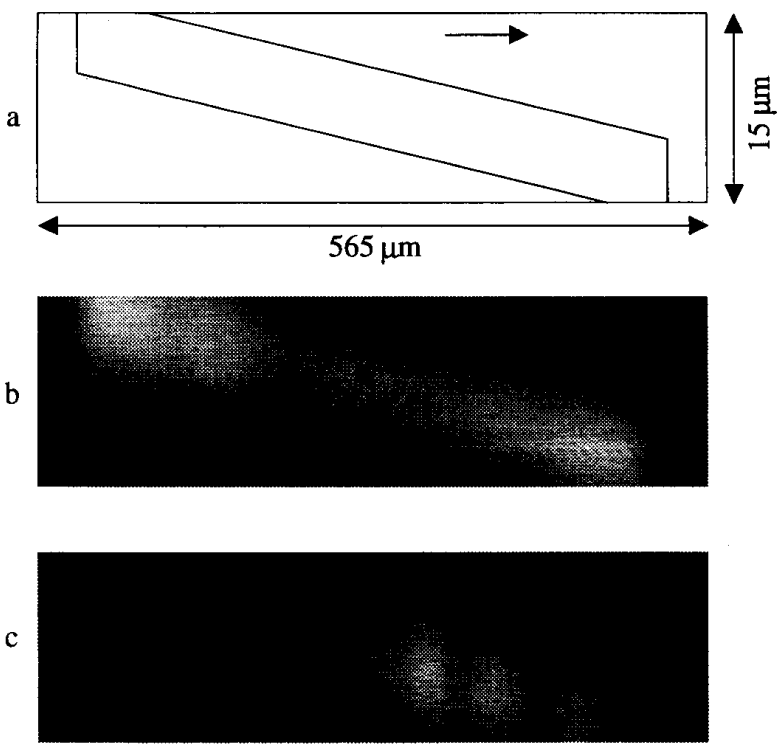

Fig. 4. a)-Sketch of the $500 \times 5 \mu \mathrm{m}^{2}$ large junction. The arrow indicates the scan direction in b) and c). b)-Voltage responce from the junction when biased in the subgap regime at $V=2.4 \mathrm{mV}$. c)Voltage response with the junction biased at $V=700 \mu \mathrm{m}$ with an applied magnetic field.

\section{CONCLUSION}

We have constructed a novel cryogenic scanning laser microscope well suited for experiments on Josephson junction circuits. The interference from electromagnetic noise and mechanical vibrations is sufficiently small. Experiments performed on various kinds of long Josephson junctions operated in the static as well as in the dynamic state have been given.

\section{ACKNOWLEDGMENT}

B. F. Jørgensen, Electromagnetic Institute is acknowledged for help with the fiber preparation, and K. Brebøl, Ferroperm Ltd. for support of and information about piezocheramic materials. Finally we thank the Optics Group at the Physics Department for fruitfull discussions and advice.

\section{REFERENCES}

[1] Yu. Ya. Divin, F. Ya. Nad, V. Ya. Pokrovski and P. M. Shadrin, "Laser probing of high-Tc superconducting thin films," IEEE Trans. on Magn., vol. 27, pp. 1101-1104, March 1991.

[2] D. Quenter, S. Stehle, T. Doderer, C. A. Krulle, R. P. Huebener, F. Mueller, J. Niemeyer, R. Popel, P. Weimann, R. Ruby, and A. T. Barfknecht, "Spatially resolved studies of the microwave properties of superconducting devices," $\mathrm{Appl}$. Phys. Lett., vol. 63, pp. 2135-2137, October 1993.
[3] R. Gross and D. Koelle, "Low temperature scanning electron microscopy of superconducting thin films and Josephson junctions," Rep. Prog. Phys., vol. 57, 651, 1994.

[4] J. Holm and J. Mygind, "A novel cryogenic Scanning Laser Microscope tested on Josephson tunnel junctions," unpublished.

[5] J. R. Lhota, M. Scheuermann, P. K. Kuo and J. T. Chen, "Observation of vortex structures in Josephson junctions by laser scanning," IEEE Trans. on Magn., vol. 19, pp. 10241026, May 1983.

[6] R. Gross, J. Bosch, H.-G. Wener, J. Fischer and R. P. Huebener, "Temperature stabilized sample stage for the investigation of high $\mathrm{Tc}$ superconductors by scanning eletron microscopy," Cryogenics, vol. 29, pp. 716-719, July 1989

[7] T. Doderer, H.-G. Wener, R. Moeck, C. Becker and R. P. Huebener," Cryogenics, vol. 30, pp. 65-67, January 1990.

[8] Yu. Ya. Divin and P. M. Shadrin, "Imaging of electrical inhomogeneities in YBCO thin-film structures by roomtemperature laser scanning microscopy," Physica $C$, vol. 232, pp. 257-262, 1994 .

[9] A. V. Ustinov, T. Doderer, R. P. Huebener, J. Mygind, V. A. Oboznov, and N. F. Pedersen, "Multi-fluxon effects in long Josephson junctions" IEEE Trans. on Appl. Supercond., vol. 3, pp. 2287-2294, March 1993.

[10] A. Rothwarf and B. N. Taylor, "Measurement of recombination lifetimes in superconductors," Phys. Rev. Lett., vol. 19, pp. 27-30, July 1967.

[11] N. E. Glass and D. Rogovin, "Transient electrodynamic response of thin-film superconductors to laser radiation" Phys. Rev., vol. B 39, pp. 11327-11344, June 1989.

[12] H. Pavlicek, L. Freytag, H. Seifert and R. P. Huebener, "Resolution limit due to thermal effects in low-temperature scanning electron microscopy," J. Low Temp. Phys., vol. 56, pp. 237$257,1984$.

[13] J. Holm, "A cryogenic scanning laser microscope for spatially resolved investigations of Josephson junction," $\mathrm{Ph}$. D. Thesis, Physics Department, Technical University of Denmark, October 1994.

[14] V. P. Koshelets, A. V. Shchukin, S. V. Shitov, and L. V. Filippenko, "Superconducting millimeter wave oscillators and SIS mixers intergrated on a chip," IEEE Trans. on Appl. Supercond., vol. 3, pp. 2524-2527, March 1993.

[15] Y. M. Zhang, D. Winkler, and T. Claeson, "Linewidth measurements of Josephson flux-flow oscillators in the band 280$330 \mathrm{GHz}$," Appl. Phys. Lett., vol. 62, pp. 3195-3197, June 1993.

[16] J. Mygind, V. P. Koshelets, A. V. Shchukin, S. V. Shitov and I. L. Lapytskaya, "Properties of Autonomous and Injection locked Flux-flow oscillators," IEEE Trans. on Appl. Supercond., in press. 